\title{
DISEÑO DE UN PROGRAMA DE APOYO A LA CALIDAD DE VIDA DE FAMILIAS CON JÓVENES Y ADULTOS CON DISCAPACIDAD INTELECTUAL
}

\section{Construction of a Family Quality of Life Support Program for families of young and adults with intellectual disability}

Natxo MarTínez Rueda

Universidad de Deusto. Facultad de Psicología y Educación. Departamento de Pedagogía Social $y$ Diversidad

Almudena FernándeZ GonZÁLEZ

Universidad de Deusto. Facultad de Psicología y Educación. Departamento de Pedagogía Social y Diversidad

José Ramón Orcasitas García

EHU-Universidad del País Vasco. Departamento de Didáctica y Organización Escolar

Delfín Montero Centeno

Universidad de Deusto. Facultad de Psicología y Educación. Departamento de Pedagogía Social y Diversidad. Avda. de las Universidades, 24. 48007 Bilbao

dmontero@deusto.es

María Villaescusa Peral

Universidad de Deusto. Facultad de Psicología y Educación. Departamento de Pedagogía Social y Diversidad

Recepción: 24 de noviembre de 2015

Fecha de aceptación definitiva: 4 de agosto de 2016 
ResumeN ${ }^{1}$ : Este artículo se fundamenta en las visiones actuales de la Calidad de Vida Familiar $(\mathrm{CdVF})$ y la intervención centrada en la familia para realizar propuestas que permitan conectar la evaluación de la CdVF con la mejora de la misma. Se describe una parte del proceso seguido en la elaboración y aplicación de un 'Programa de Apoyo a la Calidad de Vida Familiar' en el marco del servicio SAIOA-BBK, un Servicio de Información, Orientación y Apoyo a personas con discapacidad y sus familias en Bizkaia, perteneciente a Gorabide. Se presenta el proceso colaborativo entre profesionales del servicio e investigadores universitarios para construir-experimentar un programa que incida en la calidad de vida de familias con hijos o hijas jóvenes y adultos con discapacidad intelectual y se presentan sus características, resultado de su aplicación a un grupo piloto de familias $(n=5)$.

Palabras clave: calidad de vida familiar; intervención centrada en la familia; programa; empoderamiento; rutinas familiares; investigación-acción; formación de profesionales.

AвSTRACT: This paper is grounded on current conceptions on Family Quality of Life (FQoL) and family-centered intervention. It describes a part of the building process of a 'Program for Supporting Family Quality of Life', within the SAIOA-BBK frame -a Gorabide's information, guidance and support service for people with intellectual disability and their families. A major goal of this project is making proposals for professionals to fit the link between FQoL assessment and its improvement. The program was developed, constructed and tested through collaborative methods between professionals and university researchers, aiming to an increase of FQoL of families with sons or daughters among the youth and adulthood period. Program features, and how it was experimented in a pilot sample of families $(n=5)$ is presented.

KEYWORDS: family quality of life; family-centered intervention; program; empowerment; family routines; action-research; professional training.

\section{Introducción: Calidad de vida familiar y enfoque centrado en la familia}

I

OS IMPORTANTES CAMBIOS OPERADOS en la última parte del siglo XX y principios del XXI en las ciencias sociales apuntan, al menos, en una doble dirección. Por una parte, los contextos, y particularmente las interacciones sociales que se producen en ellos, son el eje central de los procesos de desarrollo personal y social (Bronfenbrenner, 1987). Por otro lado, los sujetos con derechos y con capacidad de lenguaje y acción han pasado a ocupar un lugar central en la construcción de esos contextos, convirtiéndose en interlocutores imprescindibles en el diseño de las políticas y las prácticas sociales, en una clave necesariamente dialógica (Flecha, Gómez y Puigvert, 2001).

1 Este trabajo, en el marco de un proyecto de investigación más amplio, se ha desarrollado con el apoyo económico de la Diputación Foral de Bizkaia en el marco del proyecto Bizkailab durante los años 2012, 2013, 2014 y 2015. 
El campo de la discapacidad ha avanzado en esta misma dirección. La participación de las personas con discapacidad en los diferentes contextos sociales y en el diseño de la respuesta a sus necesidades se ha convertido en uno de los ejes articuladores de las actuaciones en esta materia. La investigación y las prácticas centradas en la calidad de vida, la inclusión social o la planificación centrada en la persona son una clara muestra de ello. Teniendo en cuenta estos elementos, es lógico que se esté revalorizando el papel de la familia como contexto natural y como fuente central de apoyos, pasando además a jugar un papel determinante, junto con las propias personas con discapacidad, en la planificación de los servicios y apoyos (Brown, 2003; Córdoba, Gómez y Verdugo, 2008).

\section{Calidad de Vida Familiar}

Aunque la Calidad de Vida individual ha experimentado un gran desarrollo, tanto en la investigación, como en la práctica, la introducción del concepto de CdVF es más reciente. Mientras que existe una considerable literatura sobre la Calidad de Vida individual en personas con discapacidad, la conceptualización de la CdVF adolece de suficiente desarrollo teórico (Wang y Kober, 2011). A pesar de que se han desarrollado diferentes instrumentos para su evaluación (Hoffman et al., 2006; Isaacs et al., 2007; Giné et al., 2013; Fernández, Martínez y Orcasitas, 2012; Martínez, Fernández y Orcasitas, 2012), su aplicación práctica es casi inexistente (Zuna et al., 2009).

Esto ha llevado a realizar algunos esfuerzos por construir un modelo teórico de la CdVF (Summers, 2005; Zuna, Summers, Turnbull, Hu y Xu, 2010). A continuación, se recogen aportaciones diversas que sintetizan algunos de estos esfuerzos de consenso.

\section{a. Familia}

Clarificar el concepto de familia es relevante de cara a delimitar en qué consiste la CdVF. En este sentido, estamos de acuerdo con la definición que propone que "una familia incluye a las personas que piensan en sí mismas como parte de la familia, ya estén relacionadas por sangre o matrimonio o no, y que se apoyan entre sí de forma regular" (Poston et al., 2004: 38). Más allá de esta definición, es relevante recoger las aportaciones de la teoría de los sistemas familiares (Leal, 2008) y concebir cada familia como un sistema social dinámico y complejo con sus propias características específicas (Schneider, Wedgewood, Llewellyn, y McConnell, 2006). Una familia no se puede reducir a las características o experiencias de sus miembros, sino que conforma una totalidad en la que las interacciones entre ellos crean una red de interdependencia con su propia estructura y subsistemas, roles, reglas y patrones que caracterizan el funcionamiento familiar en un determinado momento y que le permiten mantener un equilibrio u homeostasis. La identificación de esos patrones o pautas de interacción - prácticas o rutinas relacionales- nos resultan claves para comprender el sistema familiar (Larson, Miller-Bishoff, Vauclair y Ferreira, 2014). También, y en coherencia con 
el modelo ecológico, los sistemas familiares son sistemas abiertos en interacción con otros contextos sociales más amplios, que van evolucionando y reajustándose para adaptarse a los diferentes cambios que se producen a lo largo del ciclo vital. En esta línea, las relaciones con otros entornos (familiares, de amistades, servicios, empleo o comunidad...) son también determinantes para comprender e intervenir en los sistemas familiares. Este enfoque sistémico-ecológico utilizando rutinas familiares como uno de los articuladores de la intervención ha mostrado su eficacia no solo en discapacidad intelectual (Wilder y Granlund, 2015; Holloway, Domínguez-Pareto, Cohen y Kuppermann, 2014; Larson, Miller-Bishoff, Vauclair y Ferreira, 2014), sino también en familias con enfermos crónicos, tal como muestra la revisión de literatura realizada por Crespo, Santos, Canavarro, Kielpikowski, Pryor y Féres-Carneiro (2013).

\section{b. Definiendo la CdVF y sus componentes}

Zuna et al. (2010: 262) parten de que la CdVF: a) depende de la impresión subjetiva de satisfacción de sus miembros; b) refleja en qué medida la familia como unidad es capaz de satisfacer las necesidades de sus miembros; c) La familia como totalidad tiene características que no pueden ser descritas solamente atendiendo a los miembros individuales, de forma que su calidad de vida tiene características diferenciales.

En base a estos planteamientos, se puede definir la CdVF (Zuna et al., 2010) como: “... un sentido dinámico de bienestar familiar, definido de forma colectiva y subjetiva e informado por sus miembros, en el que las necesidades a nivel individual y familiar interactúan" (p. 262), enfatizando la idea de explorar las percepciones y dinámicas familiares como un conjunto.

Este concepto de CdVF reconoce la capacidad de adaptación positiva y fortalezas de las familias con hijos o hijas con discapacidad. Además, comprende el concepto de discapacidad en su contexto, reconociendo la interacción entre las necesidades de apoyo y los recursos disponibles (Chiu, Kyzar, Zuna, Turnbull, Summers y Aya, 2013).

Construir un modelo teórico sobre CdVF supone no sólo identificar la calidad de vida como un resultado -que podemos evaluar-, sino también identificar las variables que inciden en la misma. En su revisión de la literatura, Zuna et al. (2010) agrupan las diferentes variables en cuatro grandes apartados:

- Familia como totalidad (características familiares, dinámicas familiares y variables demográficas).

- Miembros de la familia como individuos (características de los miembros y creencias).

- Servicios, apoyos e interacciones que la familia o sus miembros reciben.

- Conceptos sistémicos (sistemas, políticas y programas).

Estas variables, en la práctica, configuran una red de círculos superpuestos en el que, a modo de engranaje, las diferentes variables interactúan de forma compleja. 
Desde nuestro punto de vista, y aunque el modelo no lo señala explícitamente, hay que enfatizar los aspectos de Acciones y/o Rutinas de las familias (McWilliam, Casey y Sims, 2009). Lo mismo que en los componentes relacionados con la respuesta de los servicios y apoyos se resaltan las acciones, las 'realizaciones' de los mismos con relación a las familias e individuos, dentro de la dinámica familiar (Park et al., 2003) pondremos en primer plano las acciones habituales, actuaciones o rutinas de la familia que reflejan y fijan, desde su historia, su 'capacidad de acción’ y su forma práctica de responder a las necesidades de sus miembros y de articular su vida en común.

Zuna et al. (2010) resumen su modelo teórico de la siguiente manera:

Sistemas, políticas y programas impactan indirectamente en apoyos, servicios y prácticas a nivel individual y familiar; las características personales, demográficas y las creencias individuales junto con las características y dinámicas familiares son predictores directos de la CdVF y también interactúan con los apoyos, servicios y prácticas a nivel individual y familiar para predecir la CdVF. Solos o combinados los predictores del modelo implican un resultado en términos de calidad de vida que produce nuevas fortalezas, necesidades y prioridades familiares, que conforman nuevas entradas en el modelo, dando continuas retroalimentaciones a lo largo de la vida (p. 269).

En síntesis, podemos señalar que serán las prácticas las que condicionen su percepción y, por lo tanto, su comprensión subjetiva de CdVF. La familia vivirá y se percibirá mejor, con mayor calidad de vida, en la medida que se faciliten mejoras en esas prácticas cotidianas. En definitiva, una de nuestras hipótesis centrales para la intervención en este ámbito radica en que, mejorando las rutinas cotidianas de la familia y los hechos de interacción práctica que las acompañan, podemos mejorar sus percepciones sobre su propia calidad de vida.

Aunque en la actualidad no existe una teoría completa sobre la CdVF y resulta necesario seguir investigando sobre sus componentes y las diferentes propuestas vinculadas a los instrumentos de evaluación, incorporando perspectivas diversas (Hu, Summers, Turnbull y Zuna, 2011; Park et al., 2003; Summers, 2005; Werner et al., 2009), existen estudios que concluyen unas dimensiones de CdVF ampliamente coincidentes en la comunidad científica (Giné et al., 2013; Hoffman, Marquis, Poston, Summers y Turnbull, 2006; Brown, Brown et al., 2006; Aznar y Castañón, 2005).

La propuesta que tomamos como referencia en este trabajo, la Escala de Calidad de vida Familiar para familias con hijos/as mayores de 18 años -ECdVF +18- (Fernández et al., 2012; Giné et al., 2013; Martínez et al., 2012), identifica las siguientes dimensiones: Bienestar emocional, Interacción familiar, Salud, Bienestar económico, Organización y habilidades parentales, Acomodación de la familia, Inclusión y participación.

\section{Prácticas centradas en la familia}

El modelo centrado en la familia fue formulado en los años 80 en el ámbito de la atención temprana, trasladándose luego a otros momentos del ciclo vital(Kim y Turnbull, 2004). Hace referencia a un conjunto de creencias, valores, principios y prácticas 
orientadas a dar apoyo y fortalecer la capacidad de las familias para promover el desarrollo y aprendizaje de sus hijos (Dunst, 2002).

Aunque existen diferentes aproximaciones a este enfoque, existe un consenso acerca de sus elementos centrales (Allen y Petr, 1998; Murphy, Lee, Turnbull y Turbiville, 1995):

- La familia como unidad de apoyo: es el conjunto de la familia, definida por sí misma, quien recibe y presta el apoyo y los servicios y no sólo la persona con discapacidad.

- La familia toma las decisiones, enfatizando su protagonismo a la hora de formular sus necesidades, establecer las prioridades y definir las acciones. De ello se deduce que se hace necesario disponer de espacios y momentos de conciencia colectiva sobre la orientación deseable del cambio y cierto consenso sobre sus valores, preferencias, intereses, etc.

Por su parte, la perspectiva del empoderamiento enfatiza, en esta misma línea, las fortalezas de la familia, su competencia y capacidad de tomar decisiones, apoyando que experimenten control de sus vidas y desarrollen acciones para conseguir lo que quieren y necesitan (Turnbull, Turnbull, Erwin y Soodak, 2006; Verdugo, 2000). Esta perspectiva centrada en la familia habla de capacitación/empoderamiento como proceso y de CdVF como resultado (Turnbull, 2003). El elemento central de la capacitación gira en torno a la creación de oportunidades para que la familia pueda actuar para conseguir lo que quiere y necesita, mejorando así su funcionamiento y percepción de control (Dunst, Trivette y Deal, 1988).

Actualmente disponemos de suficiente evidencia empírica que muestra que el tipo de modelo relacional que se establece con las familias (centrado en el profesional, familia como aliado enfocado a la familia o centrado en la familia) tiene gran impacto en los sentimientos de control y autoeficacia, satisfacción, conducta parental o valoración de la utilidad de los programas (Dunst, Trivette y Hamby, 2007; EspeSherwindt, 2008), por encima de las características de los miembros de las familias o su estatus socioeconómico. Además, dentro del enfoque centrado en la familia, se pueden diferenciar las prácticas de apoyo relacionales o participativas (Dunst, Boyd, Trivette y Hamby, 2002). Las primeras se configuran en torno a las competencias de comunicación como escucha, empatía, aceptación y honestidad, mientras que las prácticas participativas se orientan a la toma de decisiones y al desarrollo de cursos de acción. Aunque ambas contribuyen al empoderamiento de las familias, las prácticas participativas tienen una relación más fuerte con los sentimientos de control de los padres y madres (Dunst y Dempsey, 2007).

Apoyándonos en Van Haren y Fiedler (2008) podemos identificar las estrategias clave para apoyar y empoderar a las familias:

- Considerar y aceptar el punto de vista de las familias, a través de la escucha y la empatía.

- Respetar los diferentes ritmos de las familias y las modalidades y estilos preferentes de participación.

- Reconocer a las familias como expertos y construir a partir de las fortalezas familiares. 
- Valorar y apoyar la toma de decisiones por parte de la familia.

- Implicar a las familias en comunicaciones abiertas, tanto en la propia familia, como con los servicios, utilizando canales y formatos diversos.

- Considerar y fortalecer la red de apoyos y servicios tanto informales como formales.

- Reconocer y celebrar los éxitos y logros de las familias.

- Orientarse a la mejora del sentido de autoeficacia de las familias.

- Desarrollar competencias y capacidades familiares.

- Promover y animar la participación de la persona con discapacidad en los encuentros y reuniones.

- Promover y abrir expectativas de futuro.

- Ayudar a las familias a articular una visión del futuro.

\section{El enfoque centrado en la familia en las prácticas de nuestro contexto}

A pesar de que hace tiempo que el enfoque centrado en la familia se ha identificado como una práctica deseable en los servicios, en nuestro contexto son escasos los trabajos en los que se muestra su desarrollo práctico. Aunque empieza a difundirse y debatirse su nivel de desarrollo en atención temprana (García-Sánchez, 2014), apenas hay referencias en otros momentos del ciclo vital. En este sentido, es relevante reconocer que aunque el papel de las familias de las personas con discapacidad ha cambiado sustancialmente en las últimas décadas, pasando a ocupar un lugar más central en la prestación de servicios (Martinez et al., 2012), no hemos encontrado en la literatura en castellano modelos sistemáticos de puesta en práctica del modelo centrado en la familia. Baste, por ejemplo, señalar que entre las buenas prácticas de Plena InclusiónFEAPS, aunque gran parte de ellas giran en torno a la centralidad de la persona y la familia, hay escasas experiencias de prácticas centradas en la familia. Otra muestra de ello es que uno de los documentos más referenciados en el ámbito (Leal, 2008) es de 1999 y traducido al castellano en 2008.

Creemos, en consecuencia, que aunque la 'filosofía' del apoyo centrado en la familia ha ido ocupando un lugar de referencia en nuestra representación de la intervención en discapacidad, se precisa de programas y experiencias concretas que desarrollen ese enfoque desde sus planteamientos más generales a sus aplicaciones prácticas para garantizar su desarrollo futuro.

En el caso de personas con discapacidad jóvenes y adultas, esta limitación se acrecienta. En parte porque en la edad adulta la referencia central se sitúa en los procesos de Planificación Centrada en la Persona (PCP). De hecho, algunos autores (Kim y Turnbull, 2004) consideran que el enfoque de planificación centrado en la familia (PCF) debe sustituirse en la edad adulta por el enfoque centrado en la persona. En este aspecto discrepamos, ya que aunque la PCP recoge en buena medida los planteamientos de la PCF, también es cierto que en la medida que la unidad familiar sigue siendo en la edad adulta la fuente de apoyo principal, parece lógico considerar a la familia también como destinataria y generadora de algunos apoyos. Si, además, las 
familias afrontan situaciones de cambio, reajuste y adaptación ante los diferentes sucesos vitales, parece de interés diseñar programas y servicios que, desde un enfoque centrado en la familia, se orienten a la CdVF.

\section{Planteamiento de la investigación}

El proyecto desarrollado ha pretendido dar un paso adelante en la investigación en CdVF, vinculando los resultados obtenidos a través de la Escala de Calidad de Vida Familiar (ECdVF) (Fernández et al., 2012; Martínez et al., 2012) con el desarrollo de procesos de intervención y acompañamiento centrados en las familias. Hemos querido, por tanto, avanzar desde la evaluación a la intervención, a través del diseño, implementación y evaluación de un proceso de intervención con familias para la mejora de su calidad de vida. Se trata, por tanto, de facilitar un proceso que le permita a la familia, a partir de su situación actual, identificar algunos resultados que quiere conseguir como familia y de diseñar un plan para lograrlos.

La PCF, entendida como un enfoque amplio, puede ser aplicada en diferentes situaciones. En consecuencia, para su concreción requiere delimitar tanto el contexto en el que va a ser aplicada como su metodología (fases, profesionales, herramientas).

Aunque en alguna medida todos los servicios y apoyos destinados a las personas con discapacidad intelectual impactan en las familias, hay un tipo de servicios que se dirigen principalmente a ellas. Por ejemplo, FEAPS-Plena Inclusión (2009: 9) define la misión del 'Servicio de Apoyo a Familias' como “Acompañar a las familias de personas con discapacidad intelectual en su proyecto de vida, partiendo de sus propios recursos y fortalezas, atendiendo a sus necesidades y demandas y facilitando los apoyos necesarios para mejorar su calidad de vida y el bienestar de cada uno de sus miembros". Entre los productos y apoyos que se prestan podemos destacar aquellos que permiten de forma más directa la incorporación del enfoque centrado en la familia:

- Orientación: se acompaña a la familia ofreciendo asesoramiento para la toma de decisiones, resolución de conflictos y la movilización de los recursos propios y del entorno, así como en su propio proceso de planificación de apoyos.

- Plan de apoyo a la familia: se ofrece la posibilidad de que aquellas familias que lo deseen puedan demandar apoyo de forma más específica e individual; para ello se elabora un Plan de Apoyo entre los profesionales del servicio y la propia familia a partir de la valoración conjunta de las necesidades de ésta (Plena Inclusión-FEAPS, 2009: 17).

En nuestro contexto próximo, Gorabide (Bizkaia), principal entidad que presta servicios a las personas con discapacidad intelectual y sus familias en Bizkaia, dispone de un Servicio de Acogida, Información, Orientación y Apoyo -SAIOA-BBK-2,

2 El servicio cuenta con cinco trabajadoras sociales, que atienden a las personas con discapacidad intelectual y a sus familias o personas tutoras, así como a las entidades públicas y privadas de Bizkaia. Su distribución se concreta en cinco zonas que agrupan varias poblaciones en base a criterios geográficos. El servicio también cuenta, entre otros, con un psicólogo, una administrativa y una persona responsable de la dirección del servicio. 
donde la atención a las familias tiene un papel central. En colaboración con Gorabide, y una vez presentados los antecedentes y sentido del proyecto, se decide que SAIOABBK puede ser el marco adecuado para poner en marcha el proyecto de investigación.

\section{Objetivos}

Este trabajo se enmarca en un proyecto más amplio que tiene como objetivo general diseñar, implementar, evaluar y generalizar un programa-proceso de intervención para la mejora de la CdVF que pueda ser incorporado en un futuro a la oferta de servicios de una entidad.

Algunas de las preguntas que han orientado el proceso de trabajo han sido:

- ¿Se pueden desarrollar acciones específicas para mejorar la CdVF?

- ¿Es posible incidir en la CdVF de familias de hijos jóvenes y adultos con discapacidad intelectual utilizando el enfoque centrado en la familia?

- ¿Tiene sentido un servicio 'nuevo' dirigido a la mejorar la de la CdVF?

- ¿Los servicios de información y orientación a familias pueden incorporar procedimientos formales de planificación centrada en la familia?

- ¿Hasta qué punto se dan prácticas centradas en la familia en los servicios actuales de información y orientación?

- ¿Un proceso de acompañamiento a lo largo de un periodo de tiempo concreto puede producir impacto en el empoderamiento de las familias y en la CdVF?

- ¿Podemos avanzar en la comprensión de la CdVF?

Una primera parte del trabajo ha consistido en el diseño del programa en un proceso colaborativo entre profesionales, familias e investigadores. En esta fase, los objetivos que han orientado el proceso han sido:

a. Desarrollar un modelo conceptual de mejora de la CdVF que sirva de referencia al desarrollo de un programa concreto de intervención en el contexto de un Servicio de Información, Orientación y Apoyo a familias.

b. Construir un programa de apoyo a la CdVF para ser aplicado en una secuencia espacio-temporal concreta.

c. Aplicar ese programa en un grupo de 5 familias para, valorando su impacto, ajustar su metodología.

d. Elaborar un manual de intervención que sirva de guía en la generalización del programa.

\section{Enfoque metodológico}

El objetivo de esta investigación no se centra solamente en el diseño de un programa de intervención con familias que pudiera ser aplicado, sino que trata de construir un programa de intervención con familias desde el contexto en el que tiene que ser aplicado, asegurando, de esta forma, el ajuste del programa a la realidad concreta y 
aumentando la probabilidad de generalización. En consecuencia, una metodología de investigación-acción participativa es la que parece adecuada para este planteamiento.

El proyecto se ha desarrollado como un proceso de colaboración con Gorabide a través de su Servicio SAIOA-BBK, que ha sido el marco en el que se ha diseñado, implementado y evaluado el programa.

La elaboración del programa se ha realizado a través de un proceso de diálogo entre investigadores y profesionales, quienes, incorporando respectivamente las aportaciones teóricas y el conocimiento práctico, han diseñado un proceso de intervención que es contrastado con las propias familias, lo que ha permitido introducir cambios y mejoras en función de sus valoraciones y recomendaciones, recogidas de forma implícita en el proceso de intervención y de forma explícita al finalizar el mismo. En este sentido, podemos hablar de diferentes niveles organizativos

1. Grupo base de investigadores: 5 Investigadores de dos universidades (Universidad de Deusto y Universidad del País Vasco) con las funciones de orientar el trabajo desde sus bases teóricas, fundamentar las propuestas, hacer el seguimiento del trabajo facilitando cambios comprensivos y operativos dialogados en el Equipo del Seminario Mixto y garantizar la recogida de datos de la investigación.

2. Grupo base de profesionales: 5 Profesionales de Gorabide con responsabilidades en la atención a familias, con las funciones de analizar las propuestas y ajustarlas a las posibilidades y recursos de la entidad, así como de poner en práctica y avanzar en las propuestas con las familias y recoger los datos.

3. Puesta en práctica y contraste con las familias: La implementación del programa con las familias permite someter a su valoración el programa diseñado y tomar decisiones acerca de los cambios y mejoras que haya que introducir.

4. Seminario de Investigadores y profesionales con la función de realizar un seguimiento de la implantación del programa y del impacto en las familias y tomar decisiones sobre la introducción de los ajustes y adaptaciones necesarias sobre las acciones a realizar y su adecuación a las familias, los materiales, la recogida de la información y el seguimiento.

La forma de funcionamiento ha consistido en el establecimiento de un esquema de trabajo en el que desde los dos grupos base se hacían aportaciones que se consensuaban en el seminario y que, posteriormente, se contrastaban con las familias en el propio proceso de intervención. Este planteamiento tiene por objeto garantizar tanto los elementos teóricos de un programa de intervención centrado en la familia, como su ajuste a un contexto concreto.

Las fases seguidas en la elaboración del proyecto se describen en el Cuadro 1.

\begin{tabular}{|l|l|}
\hline \multicolumn{2}{|c|}{ CUADRO 1. Fases de desarrollo del proyecto } \\
\hline Pasos & \multicolumn{1}{|c|}{ Descripción } \\
\hline 1 & $\begin{array}{l}\text { Configuración del equipo mixto de investigación y criterios de selección de las familias } \\
\text { participantes }\end{array}$ \\
\hline 1.1 & $\begin{array}{l}\text { Redacción y acuerdo del proyecto y firma del convenio de colaboración Gorabide- } \\
\text { Universidad de Deusto }\end{array}$ \\
\hline
\end{tabular}

(C) Ediciones Universidad de Salamanca / CC BY-NC-ND

Siglo Cero, vol. 47 (3), n. ${ }^{\circ} 259,2016$, julio-septiembre, pp. 47-67 
DISEÑO DE UN PROGRAMA DE APOYO A LA CALIDAD DE VIDA DE FAMILIAS CON JÓVENES

Y ADULTOS CON DISCAPACIDAD INTELECTUAL

N. MARTÍNEZ, A. FERNÁNDEZ, J. R. ORCASITAS, D. MONTERO Y M. VILLAESCUSA

\begin{tabular}{|c|c|}
\hline \multicolumn{2}{|r|}{ CuADro 1. Fases de desarrollo del proyecto } \\
\hline Pasos & Descripción \\
\hline 1.2 & $\begin{array}{l}\text { Selección de los profesionales participantes en el estudio }(\mathrm{N}=5) \text { y del Servicio desde el } \\
\text { que se va a desarrollar la intervención con familias. }\end{array}$ \\
\hline 1.3 & $\begin{array}{l}\text { Establecimiento de los criterios y selección de la muestra }(\mathrm{N}=5) \text {, tomando como referen- } \\
\text { cia que sean familias con hijos en edades de transición a la vida adulta }(16-25) \text {. }\end{array}$ \\
\hline 2 & $\begin{array}{l}\text { Construcción de equipo y de referencias compartidas: Revisión de las referencias teó- } \\
\text { ricas para la evaluación e intervención con familias }\end{array}$ \\
\hline 2.1 & $\begin{array}{l}\text { Revisión bibliográfica y estado de la cuestión de: CdVF, variables relacionadas, Evalua- } \\
\text { ción de la CdVF y Modelo de Intervención Centrado en la Familia }\end{array}$ \\
\hline 2.2 & Redacción de Documento síntesis de referencia \\
\hline 2.3 & $\begin{array}{l}\text { Debate en contraste con el conocimiento profesional y aprobación de documento de } \\
\text { referencia }\end{array}$ \\
\hline 3 & $\begin{array}{l}\text { Construcción de equipo y referencias compartidas: Revisión de referencias prácticas } \\
\text { en la evaluación e intervención con familias }\end{array}$ \\
\hline 3.1 & $\begin{array}{l}\text { Análisis de programas y servicios de Gorabide con relación a las familias: enfoque, tipo- } \\
\text { logía de programas, herramientas }\end{array}$ \\
\hline 3.2 & $\begin{array}{l}\text { Análisis de caso tipo de intervención desde el servicio SAIOA: contraste con referentes } \\
\text { teóricos de CdVF }\end{array}$ \\
\hline 3.3 & $\begin{array}{l}\text { Evaluación de la CdVF a través de la EdCVF (referencia): autoaplicación, análisis de la } \\
\text { herramienta, aportaciones y limitaciones del instrumento }\end{array}$ \\
\hline 4 & Diseño del Programa de Apoyo a la CdVF centrado en la familia \\
\hline 4.1 & Clarificación del encuadre de la propuesta: destinatarios, espacios y tiempos \\
\hline 4.2 & $\begin{array}{l}\text { Elaboración de dos propuestas de programa: a) desde la práctica, tratando de avanzar; b) } \\
\text { desde la teoría, tratando de ajustarse a los condicionamientos y posibilidades del servicio }\end{array}$ \\
\hline 4.3 & $\begin{array}{l}\text { Diseño de una propuesta de síntesis: manual de la experiencia piloto (objetivos, fases y } \\
\text { herramientas) }\end{array}$ \\
\hline 5 & Ensayo y seguimiento de la aplicación del programa en experiencia piloto \\
\hline 5.1 & Selección de familias \\
\hline 5.2 & Presentación y análisis del desarrollo del programa \\
\hline 5.3 & Evaluación del programa y contraste con las familias \\
\hline 6 & Elaboración del programa definitivo \\
\hline 6.1 & Identificación de mejoras a introducir desde la experiencia de los profesionales \\
\hline 6.2 & Actualización de las referencias teóricas del programa \\
\hline 6.3 & Contraste con otros profesionales del Servicio \\
\hline 6.4 & Incorporación de perspectiva de las familias participantes \\
\hline 6.5 & Redacción de la versión modificada y ajuste de las herramientas \\
\hline
\end{tabular}

\section{El diseño del programa: procedimientos y herramientas}

En el marco de las fases anteriores, el procedimiento específico de diseño, ensayo y revisión del programa ha incluido los siguientes procedimientos:

- Identificación y debate de los elementos del enfoque centrado en la familia. 
- Revisión de materiales de apoyo para la elaboración de Planes de Apoyo Familiar.

- Acuerdo sobre las características espacio-temporales del programa, considerando las posibilidades de recursos del Servicio y garantizando un tiempo suficiente para el desarrollo de procesos de acompañamiento.

- Acuerdo sobre los destinatarios del programa, las personas que participan y los criterios de selección para la experiencia piloto.

- Construcción, debate y acuerdo de las propuestas del proceso de intervención, a partir de las aportaciones de los dos equipos base estableciendo una secuencia de sesiones/encuentros con la familia, considerando en cada sesión: objetivos, desarrollo de la sesión, estrategias y herramientas.

- Redacción del Manual de Apoyo, incluyendo la denominación del programa.

La construcción colectiva a lo largo de diversos encuentros recogidos en Actas ha permitido, por una parte, que el propio proceso sea formativo para todas las personas participantes y, por otra, incorporar de manera consensuada las distintas aportaciones realizadas por el equipo de investigadores de las dos Universidades, por los profesionales de Gorabide y por las familias participantes.

\section{La puesta en práctica del programa y su revisión}

El programa en su fase piloto se ha desarrollado en las siguientes fases:

A. Selección de familias participantes. A partir de las personas usuarias inscritas en Gorabide, se identifican las familias con hijos/as con edades entre 17-25 años, es decir, en fase de transición, y se determinan los requisitos mínimos para participar en el programa:

a. Que la persona con discapacidad intelectual conviva en el domicilio familiar.

b. Que la familia no esté participando ya en otros programas de formación familiar.

c. Que la familia exprese el deseo y aceptación para participar en el programa.

d. Que los miembros de la familia identificados como significativos acepten asistir regularmente a las reuniones que se establezcan, así como trabajar activamente en el plan de intervención acordado.

B. La selección de las familias participantes en la fase piloto se realiza tomando en consideración estos criterios y el conocimiento que las trabajadoras sociales tienen de cada familia y valorando su posible interés en la propuesta. De esta forma, se identifican 7 familias con las que se contacta telefónicamente para informarles e invitarles a participar en el proyecto, indicando el nivel de compromiso. De las 7 familias contactadas, 5 aceptaron participar.

C. Implementación del Programa. La puesta en práctica de la Experiencia Piloto del Programa de Acompañamiento para la Calidad de Vida Familiar comenzó en marzo de 2013 y finalizó en diciembre de 2013. 


\section{La evaluación y mejora del programa: sistematización}

La evaluación del programa en la fase piloto se ha realizado:

- Por medio de revisiones periódicas de la puesta en práctica del programa en los encuentros regulares de los equipos de trabajo que han ido analizando tanto la intervención con cada una de las familias, como su desarrollo general. Estos encuentros han quedado recogidos en las Actas correspondientes que han permitido documentar tanto la evolución de cada familia, como las valoraciones de los profesionales sobre el desarrollo del programa.

- La visión de las familias, además de las aportaciones en cada una de las sesiones, se ha recogido a través de una sesión específica de evaluación y un cuestionario de valoración de la experiencia. Las 5 familias participantes han valorado de forma positiva su participación en el programa. Añadir que también se ha utilizado la Escala de Calidad de Vida Familar CdVF+18 (Giné et al., 2013; Fernández, Martínez y Orcasitas, 2012) tanto al inicio como a la finalización del programa, con el objetivo de valorar su impacto en la calidad de vida. En este sentido, señalar que los resultados no han sido concluyentes debido a las propias características del instrumento y de la muestra.

- Por último, se ha llevado a cabo un grupo focal con los profesionales participantes en el programa para valorar sus aportaciones y debilidades y realizar propuestas de mejora.

Todo ello ha permitido al equipo introducir importantes mejoras en el programa. Por una parte, orientadas a una mayor sistematización a través de identificar con más claridad las actividades de cada fase, enfatizándolas y explicitándolas con las familias y estableciendo la indicación de ponerlas en práctica de la misma forma por parte de todos los profesionales.

Por otra parte, se han ajustado las herramientas como el principal soporte al desarrollo del programa. En este sentido, se concretan en mayor medida las herramientas a utilizar en cada fase del programa, así como las instrucciones de uso, se incorporan algunas herramientas nuevas y se consensúan las estrategias de intervención por parte de los profesionales que, de acuerdo al análisis realizado durante la fase piloto, han resultado especialmente exitosas.

\section{Resultado: un Programa de Apoyo a la Calidad de Vida Familiar}

Como resultado de todo el proceso de trabajo y una vez evaluado, incorporando la perspectiva de profesionales y familias, se ha sistematizado un Programa de Apoyo a la Calidad de Vida familiar que se resume a continuación, atendiendo, en primer lugar, a sus componentes fundamentales y, en segundo lugar, a las fases en las que se desarrolla. 


\section{Los elementos del programa}

La finalidad del programa consiste en crear un espacio de encuentro de toda la familia, para construir y poner en práctica un Plan de Mejora Familiar que incluya la perspectiva y necesidades de todos sus componentes, con el apoyo de un profesional -testigo-. Ese proceso pretende incidir en la capacitación/empoderamiento de las familias, contribuyendo a una mejora de su Calidad de Vida.

Los principales componentes del programa, configurando su estructura básica, se reflejan en el Cuadro 2, donde se resumen las bases sobre las que se construye el programa, los objetivos a los que se dirige, los resultados previstos y las estrategias de intervención.

\begin{tabular}{|c|c|c|c|}
\hline \multicolumn{4}{|c|}{ CuAdro 2. CdVF y Enfoque centrado en la familia } \\
\hline Base & $\begin{array}{c}\text { Objeto de trabajo: } \\
\text { empoderamiento familiar }\end{array}$ & $\begin{array}{c}\text { Resultados } \\
\text { CdVF }\end{array}$ & $\begin{array}{c}\text { Estrategias de } \\
\text { intervención }\end{array}$ \\
\hline $\begin{array}{l}\text { - Familia en } \\
\text { conjunto } \\
\text { - Fortalezas y } \\
\text { recursos familiares } \\
\text { - Colaboración } \\
\text { familia- } \\
\text { profesionales } \\
\text { - Redes de apoyo }\end{array}$ & $\begin{array}{l}\text { - Activación y control } \\
\text { familiar, autoeficacia } \\
\text { - Competencias } \\
\text { comunicativas } \\
\text { - Regulación de roles y } \\
\text { rutinas } \\
\text { - Decisiones y planes } \\
\text { - Coordinación de } \\
\text { acciones } \\
\text { - Reflexión y evaluación } \\
\text { - Servicios y apoyos } \\
\text { - PDI y servicios que } \\
\text { recibe }\end{array}$ & $\begin{array}{l}\text { - } \text { Bienestar } \\
\text { emocional } \\
\text { - Interacción } \\
\text { familiar } \\
\text { - Salud } \\
\text { - } \text { Bienestar } \\
\text { económico } \\
\text { - Organización } \\
\text { y habilidades } \\
\text { parentales } \\
\text { - Acomodación } \\
\text { de la familia } \\
\text { - Inclusión y } \\
\text { participación }\end{array}$ & $\begin{array}{l}\text { - Estrategias de } \\
\text { relación: escucha, } \\
\text { empatía, aceptación, } \\
\text { mirada positiva... } \\
\text { - Estrategias de } \\
\text { participación } \\
\text { (compartir } \\
\text { información, } \\
\text { animar en la toma } \\
\text { de decisiones, } \\
\text { estimular el uso de } \\
\text { sus conocimientos y } \\
\text { recursos, desarrollar } \\
\text { cursos de acción, } \\
\text { ayudar a poner en } \\
\text { práctica nuevas } \\
\text { habilidades) }\end{array}$ \\
\hline
\end{tabular}

La base de la intervención se puede resumir en las creencias y valores clave que guían la actuación profesional y que se relacionan con una mirada a la familia como totalidad, poniendo en primer plano las fortalezas y recursos familiares, enfatizando la relevancia de las redes sociales (tanto formales como informales) de apoyo. Todo ello en el marco de una relación de colaboración, en clave de igualdad y de respeto a la singularidad y perspectiva de la propia familia.

El foco de intervención se sitúa en el empoderamiento familiar para afrontar las principales cuestiones o temas de la vida familiar. Ello supone, en primer lugar, orientar la intervención a la activación y 'control' familiar. En este sentido, se entiende el trabajo con las familias como la movilización de sus recursos para afrontar sus necesidades. Un segundo aspecto tiene que ver con facilitar conversaciones efectivas en la familia a través de reconocer los diferentes puntos de vista y de facilitar contextos comunicativos 'controlados' que promuevan la expresión de inquietudes, la escucha, los acuerdos. El tercer aspecto, relacionado con los anteriores, tiene que ver con la 
capacidad de la familia para proponerse metas y planificar cursos de acción, parte de ellos vinculados a rutinas de la vida cotidiana, que se implementan a través de coordinar acciones tanto internamente, dentro de la familia, como en su relación con sus redes de apoyo. Vinculado a esos aspectos, aunque con un papel central, se sitúa el acceso a recursos y servicios, tanto para la persona con discapacidad como para el conjunto de la familia.

Los resultados tienen que ver con el 'contenido' de las preocupaciones familiares que se relacionan con las dimensiones relevantes de CdVF. Desde el punto de vista de la intervención, constituirían los 'temas a tratar', que serán aquellos que sean relevantes para cada familia. Desde el punto de vista del proceso de intervención -obviamente, no desde el punto de vista de la familia-, los temas no son lo más importante, sino la posibilidad que nos ofrecen de poner en juego el desarrollo de las competencias familiares.

Por último, para facilitar este proceso, los profesionales pondrán en juego, por una parte, estrategias de relación orientadas a la construcción de un contexto adecuado y, por otra, acciones de participación orientadas a las diferentes actuaciones que la familia vaya a desarrollar.

\section{Las fases del programa}

Como señalábamos, el programa consiste en encuentros sistemáticos y regulares de toda la familia con el apoyo de un o una profesional para afianzar sus posibilidades de éxito en sus interacciones prácticas (las acciones que realizan habitualmente tanto dentro de la familia como fuera). Ello incide en una mejora en la capacidad de acción de la familia-competencias familiares-y, en consecuencia, en su sentido del bienestar.

El programa se compone de una serie de encuentros con toda la familia precedidas de una primera fase denominada Información y Compromiso de Participación, en la que se informa a las familias, vía telefónica o presencial cuando sea necesario, de las características del programa con el apoyo de un folleto. El programa se inicia cuando una familia, por iniciativa propia o por sugerencia de algún profesional, se interesa por el programa y es informada del sentido, características e implicaciones de su posible participación. La finalidad de esta primera fase es que la familia pueda tomar una decisión informada sobre su participación.

La segunda fase, denominada Visión Familiar, tiene como finalidad que los miembros de la familia compartan entre ellos su visión acerca de cómo están, cómo les van las cosas, sus inquietudes y lo que es importante para ellos en relación al bienestar familiar. También se busca identificar los apoyos con los que cuentan y los aspectos de su funcionamiento que desean mejorar. En un primer momento, señalan lo que les gusta y valoran de los diferentes miembros de la familia y de la familia en su conjunto. Es importante que el profesional ayude a la familia a elaborar un perfil positivo del funcionamiento familiar: enfatizando lo bueno que tiene, destacando las fortalezas que tiene cada miembro de la familia individualmente considerado y la familia en su conjunto. Es importante que cada miembro de la familia perciba que tiene su espacio para 
decir lo que piensa y siente y que se sienta reconocido por los demás. Después de este primer momento, el o la profesional ayuda a la familia a pensar en su funcionamiento como familia, explorando las cosas que son importantes para ella (en su conjunto, pero también de los miembros individuales), ayudándose del ecomapa (McWilliam, 2001) para identificar con qué apoyos cuentan (tanto formales como informales) y cuáles son las cosas que funcionan y no funcionan (Sanderson y Acraman, 2004) en su desempeño cotidiano como familia. Algunos aspectos clave en esta fase son:

- Presentación y reconocimiento de cada miembro de la unidad familiar a través de compartir la información básica y de señalar lo que nos gusta o valoramos de cada persona.

- Explicitar ¿cómo es nuestra familia? ¿Qué es importante para nosotros como familia?, dos cuestiones de gran relevancia que explicitan lo que es valioso para cada familia y que es construido colectivamente en el grupo familiar.

- Fijado y consensuado lo importante-valioso, la imagen de la familia se fortalece y es capaz de señalar, además de los aspectos que en su vida cotidiana 'funcionan', las dificultades y las necesidades de apoyo que explícitamente han señalado como relevantes y que, en su percepción, 'no funcionan'.

La tercera fase, Plan de Mejora Familiar, concreta los temas o cuestiones sobre los que va a trabajar la familia y planifica las acciones que se van a realizar. Se trata de que la familia dialogue sobre las cuestiones o puntos que se han comentado en el apartado de "Funciona/No funciona" de cara a seleccionar aquellos aspectos sobre los que les gustaría proponer cambios o metas. Una vez seleccionadas las prioridades sobre las que se quieren centrar, la familia y el profesional formulan con claridad cuáles son las metas que quieren conseguir en cada una de ellas, las acciones a poner en marcha para lograrlas y los responsables de las mismas.

La finalidad de la cuarta fase, Seguimiento y apoyo, es la de hacer un acompañamiento para que se realicen las acciones propuestas en el Plan de Mejora Familiar. En este sentido, es importante prever algunos apoyos que el profesional puede tener que prestar. Aunque el seguimiento y apoyo se puede realizar de diferentes formas, ajustando tanto las posibilidades de las familias como las de los y las profesionales, pudiéndose realizar de forma presencial, telefónica, por e-mail o combinada y pudiendo participar uno o más de un miembro de la familia, es un requisito necesario que se realice al menos un encuentro presencial en el que estén presentes todos los miembros de la familia que están participando en el programa.

La última fase supone la Evaluación y cierre del proceso, en la que la familia evalúa la experiencia y analiza los avances logrados derivados de las acciones establecidas en el Plan. Se trata de valorar la experiencia describiendo los avances en los objetivos previstos y los cambios producidos en la vida familiar. Cuando se han conseguido, aunque sea parcialmente, los objetivos y nuevas rutinas se van asentando, los miembros de la familia se sienten más competentes y la familia como unidad se fortalece. En esta fase nos parece importante, también, la tarea del profesional de apoyo desde su cualificada posición de testigo para facilitar la descripción constructiva y positiva del proceso y de las nuevas rutinas vitales que van a permanecer. 
En síntesis, el proceso de colaboración con las familias consta de las siguientes fases:

A. Fase Primera: Información y Compromiso de Participación. Informar a la familia y establecer un compromiso inicial de participación.

B. Fase Segunda: Visión Familiar. Compartir información relacionada con las diferentes visiones e inquietudes de los miembros de la familia facilitando la emergencia de una narración aceptable para todos y que permita la acción deseable.

C. Fase tercera: Plan de Mejora Familiar. Consensuar con la familia los objetivos y acciones a realizar.

D. Fase Cuarta: Seguimiento y apoyo. Acompañar y apoyar a la familia en el plan de mejora familiar planificado.

E. Fase Quinta: Evaluación y cierre. Valorar la experiencia y analizar los avances realizados.

Por último, el programa incorpora un instrumento destinado a evaluar la CdVF y el impacto del programa en la CdV de la familia: las Escalas de Calidad de Vida Familiar (CdVF-E) (Giné et al., 2013) para familias con hijos con DI mayores de 18 años.

\section{Conclusiones}

En resumen, el programa de CdVF que hemos presentado consiste en encuentros sistemáticos y regulares del conjunto de la familia, con el apoyo de un testigo/profesional para afianzar sus posibilidades de éxito en su funcionamiento cotidiano, rutinas -las acciones que realizan habitualmente tanto dentro de la familia como fuera-. Ello persigue un impacto en los sentimientos de competencia y capacitación de las familias para gestionar su funcionamiento, incidiendo en su calidad de vida familiar. Tanto a través de las aportaciones de los profesionales como de las propias familias, se identifican algunos de los aspectos clave que este programa aporta y que permiten validar la conclusión de que implementa mayores sentimientos de competencia y empoderamiento familiar en la gestión de su funcionamiento como familia.

\section{La participación de toda la familia}

Desde el punto de vista de los profesionales y las familias esta es una de las principales aportaciones del programa.

Los profesionales enfatizan que juntar a todos los miembros de la familia, incluida la PDI, contribuye a:

- Mejorar la comprensión del funcionamiento familiar ya que se muestran las formas que la familia tiene de relacionarse, plantear los temas y tomar decisiones. En palabras de los profesionales: "Juntarles permite ver cosas que no han aparecido hasta ahora"; "afloran situaciones y necesidades que están latentes". Esta ampliación de la comprensión familiar ayuda a que las propuestas de acción o la toma de decisiones que se vayan planteando sean más ajustadas a la 
situación familiar y puedan ser contrastadas desde el principio. Esto incrementa sus posibilidades de éxito.

- Generar un espacio de diálogo que ayuda a identificar y a hacer compatibles las necesidades de cada miembro de la unidad familiar: "Un cambio sustancial es tener en cuenta las necesidades de todos”. La capacidad que tenga la familia de hacer compatibles las necesidades, intereses o aspiraciones de todos sus miembros es un aspecto clave a considerar a la hora de promover la calidad de vida familiar (Zuna, 2014; Fernández et al., 2015).

Las familias, por su parte, valoran la oportunidad de abordar cuestiones que les preocupan como familia pero que no encuentran el momento de tratarlas: "te obliga a hablar, a pararse"; "hemos pensado en cómo lo hacemos y hemos hablado más"; "hablar de cosas que no hemos hablado nunca". También valoran que les ayuda a sentirse más competentes como familia: "Hemos recuperado el control”; "ayuda a unificar criterios y a tomar decisiones"; "ayuda a estar más preparados cuando ocurran cosas"; "nos ha dado más seguridad"; "nos hemos sentido más fuertes".

\section{La presencia de un profesional de apoyo, 'testigo'}

El espacio de encuentro que se genera con un 'testigo' -el profesional de apoyo- en un diálogo ordenado, ayuda a la familia a sentirse acompañada y a 'activarse'. Presentarse personalmente frente a un tercero en el grupo familiar, cuando esto ha sido consentido, facilita la puesta en escena de nuevos diálogos y discursos. Diálogos que remitirán a unos contenidos de interés y apuntarán algunas maneras relacionales nuevas. Las familias valoran que hacer ese proceso con un profesional de apoyo ayuda a escucharse y asumir compromisos: "Alguien ajeno hace de pantalla, reflejo, nos ayuda, nos pregunta"; "te devuelven como nos ve”; "hacemos más caso si lo dice alguien de fuera”; "ayuda hablar con un testigo”.

\section{Un proceso sistemático en un tiempo delimitado}

Tanto los profesionales como las familias valoran positivamente que el proceso de trabajo tenga una duración limitada, que permite a las familias activarse en torno a unos objetivos compartidos. El uso de las herramientas de forma rigurosa y la realización de actividades en la secuencia que establece el programa facilitan el proceso y es uno de los aspectos que marca la diferencia en los logros que consiguen las familias. En palabras de las propias familias, "un tiempo delimitado ayuda a asentar los compromisos y no agobia”; "tener por escrito el plan nos ayuda”; "establecer los compromisos es importante porque nos compromete".

En definitiva, este programa consiste en desarrollar un proceso que le permita a la familia, a partir de su situación actual, identificar algunos resultados que quiere conseguir como familia y diseñar un plan de apoyo para lograrlos. Ese espacio compartido 
para toda la familia, con un testigo en una secuencia de acciones, puede contribuir a mejorar la calidad de vida familiar. Este programa, que en la siguiente fase de la investigación se va a ensayar con un grupo más amplio de familias para evaluar su impacto, ofrece un marco teórico sólido, una secuencia de actividades contrastadas y un conjunto de herramientas que lo facilitan.

\section{Referencias bibliográficas}

Allen, R. I. y Petr, C. G. (1998). Rethinking family-centered practice. American Journal of Orthopsychiatry, 568 (1), 4-15. doi:10.1037/h008026.of.

Aubert, A., Flecha, A., García, C., Flecha, R. y Racionero, S. (2008). Aprendizaje dialógico en la sociedad de la Información. Barcelona: Hipatia.

Aznar, A. S. y CASTAÑón, D. G. (2005). Quality of life from the point of view of Latin American families: A participative research study. Journal of Intellectual Disability Research, 49, 784-788.

Bronfenbrenner, U. (1987). Ecología del desarrollo humano. Barcelona: Paidós.

Brown, I. A. (2003). Family quality of life: Canadian results from an international study. Journal of Developmental E Physical Disabilities, 15 (3), 207-230.

Brown, I., Brown, R. I., Baum, N. T., Isaacs, B. J., Myerscough, T., Neikrug, S., ... Wang, M. (2006). Family Quality of Life Survey: Main caregivers of people with intellectual or developmental disabilities. Toronto, Canada: Surrey Place Centre.

Chiu, C., Kyzar, K., Zuna, N., Turnbull, A. P., Summers, J. A. y Aya, V. (2013). Family quality of life. En M. W. WehmeYer (Ed.), Oxford handbook of positive psychology and disability (pp. 365-392). New York, NY: Oxford University Press.

Córdoba, L., Gómez, J. y Verdugo, M. Á. (2008). Calidad de vida familiar en personas con discapacidad: un análisis comparativo. Universitas Psychologica, 7 (2), 369-383.

Crespo, C., Santos, S., Canavarro, M. C., Kielpikowski, M., Pryor, J. y Féres-Carneiro, T. (2013). Family routines and rituals in the context of chronic conditions: A review. International Journal of Psychology, 48 (5), 729-746. doi:10.1080/00207594.2013.806811.

Dunst, C. J. (2002). Family-centered practices: Birth through high school. Journal of Special Education, 36 (3), 139-147.

Dunst, C. J., Boyd, K., Trivette, C. M. y Hamby, D. W. (2002). Family-oriented program models and professional helpgiving practices. Family Relations, 51, 221-229. doi: 10.1111/j.1741-3729.2002.00221.x.

Dunst, C. J. y Dempsey, I. (2007). Family-professional partnerships and parenting competence, confidence, and enjoyment. International Journal of Disability, Development and Education, 54 (3), 305-318.

Dunst, C. J., Trivette, C. M. y Deal, A. (1988). Enabling and empowering families: Principles and guidelines for practice. Cambridge, MA: Brookline Books.

Dunst, C. J., Trivette, C. M. y Hamby, D. W. (2007). Meta-analysis of family-centered helpgiving practices research. Mental Retardation and Developmental Disabilities Research Reviews, 13, 370-378.

ESPE-SHERWINDT, M. (2008). Family-centred practice: collaboration, competency and evidence. Support for Learning, 23 (3), 136-143.

FEAPs-Plena InClusión (2009). Modelo de servicio de apoyo a familias. 2. a edición. Madrid: FEAPS. 
Fernández, A., Martínez, N. y Orcasitas, J. R. (2012). Construcción y validación de dos escalas de Calidad de Vida Familiar para familias españolas con hijos e hijas con discapacidad intelectual menores y mayores de 18 años. En D. Montero y P. Fernández de LARrinOA (Coords.), Calidad de vida, inclusión social y procesos de intervención. Bilbao: Universidad de Deusto.

Fernández, A., Montero, D., Martínez, N., Orcasitas, J. R. y Villaescusa, M. (2015). Calidad de vida familiar: marco de referencia, evaluación e intervención. Siglo Cero, (46) 2, 254, 7-29.

Flecha, R., Gómez, J. y Puigvert, L. (2001). Teoría sociológica contemporánea. Buenos Aires: Editorial Paidós.

García-SÁnchez, F. (2014). Atención temprana: Enfoque centrado en la familia. En XXIX Congreso AELFA (pp. 286-302). Madrid: AELFA.

Giné, C., Vilaseca, R., Gràcia, M., Mora, J., Orcasitas, J. R., Simón, C., Torrecillas, A. M., Beltran, F. S., Dalmau, M., Pro, M. T., Balcells-Balcells, A., Masa, J. M., AdamAlcocera, A. L. y Simó-Pinatella, D. (2013). Spanish family quality of life scales: Under and over 18 years old. Journal of Intellectual \& Developmental Disability, 38 (2), 141-148.

Hoffman, L., Marquis, J., Poston, D., Summers, J. A. y Turnbull, A. (2006). Assessing family outcomes: Psychometric evaluation of the Beach Center Family Quality of Life Scale. Journal of Marriage and Family, 68, 1069-1083.

Holloway, S. D., Domínguez-Pareto, I., Cohen, S. R. y Kuppermann, M. (2014). Whose Job Is It? Everyday routines and quality of life in Latino and non-Latino families of children with intellectual disabilities. Journal of Mental Health Research in Intellectual Disabilities, 7 (2), 104-125.

Hu, X., Summers, J. A., Turnbull, A. A. y Zuna, N. N. (2011). The quantitative measurement of family quality of life. Journal of Intellectual Disability Research, 55 (12), 1098-1114.

Isaacs, B. J., Brown, I., Brown, R. I., Baum, N., Myerscough, T. y Neikrug, S. (2007). The international family quality of life project: Goals and description of a survey tool. Journal of Policy and Practice in Intellectual Disabilities, 4, 177-185.

Kim, K. y Turnbull, A. (2004). Transition to adulthood for students with severe intellectual disabilities: Shifting toward person-family interdependent planning. Research and Practice for Persons with Severe Disabilities, 29 (1), 53-57.

Larson, E., Miller-Bishoff, T., Vauclair, C. y Ferreira, L. C. (2014). Family routines within the ecological niche: An analysis of the psychological well-being of U.S. caregivers of children with disabilities. Frontiers in Psychology, 51-14. doi:10.3389/fpsyg.2014.00495.

LEAL, L. (2008). Un enfoque de la discapacidad intelectual centrado en la familia. Madrid: FEAPS.

Martínez, N., Fernández, A. y Orcasitas, J. (2012). Calidad de vida de las familias con hijos/as con discapacidad intelectual en el País Vasco. En D. Montero y P. Fernández de LARrinOa (Coords.), Calidad de vida, inclusión social y procesos de intervención. Bilbao: Universidad de Deusto.

McWilliam, R. A. (2001). Understanding the Family Ecology. Chapel Hill, NC: Project Integrate.

McWilliam, R. A., Casey, A. M. y Sims, J. (2009). The routines-based interview: A method for gathering information and assessing needs. Infants and Young Children, 22 (3), 224-233.

Murphy, D. L., Lee, I. L., Turnbull, A. P. y Turbiville, V. (1995). Family-centered Program Rating Scale: An instrument for program evaluation and change. Journal of Early Intervention, 19 (1), 24-42.

Park, J., Hoffman, L., Marquis, J., Turnbull, A. P., Poston, D., Mannan, H., Wang, M. y Nelson, L. L. (2003). Toward assessing family outcomes of service delivery: Validation of a family quality of life survey. Journal of Intellectual Disability Research, 47 (4/5), 367-384. 


\section{DISEÑO DE UN PROGRAMA DE APOYO A LA CALIDAD DE VIDA DE FAMILIAS CON JÓVENES Y ADULTOS CON DISCAPACIDAD INTELECTUAL \\ N. MARTÍNEZ, A. FERNÁNDEZ, J. R. ORCASITAS, D. MONTERO Y M. VILLAESCUSA}

Poston, D., Turnbull, A., Park, J., Mannan, H., Marquis, J. y Wang, M. (2004). Calidad de vida familiar: un estudio cualitativo. Siglo Cero, 35 (3) 211, 31-48.

SANDERson, H. y ACraman, C. (2004). Family essential lifestyle plans. Recuperado de www.helensandersonassociates.co.uk/media/10232/family\%20essential\%20lifestyle\%20plans.pdf.

Schneider, J., Wedgewood, N., Llewellyn, G. y McConnell, D. (2006). Families challenged by and accommodating to the adolescent years. Journal of Intellectual Disability Research, 50 (12), 926-936.

Summers, J. A. (2005). Conceptualizing and measuring family quality of life. Journal of Intellectual Disability Research, 49 (10), 777-783.

Turnbull, A. P. (2003). La calidad de vida de la familia como resultado de los servicios: el nuevo paradigma. Siglo Cero, 34 (3), 207, 59-73.

Turnbull, A. P., Turnbull, H. R., Erwin, E. y SoOdak, L. (2006). Families, professionals, and exceptionality: Positive outcomes through partnership and trust ( 5 th ed.). Columbus, $\mathrm{OH}$ : Merrill/Prentice Hall.

Van Haren, B. y Fiedler, C. R. (2008). Support and empowerment families of children with disabilities. Intervention in School and Clinic, 43 (4), 231-235.

Verdugo, M. Á. (2000). Familias y Discapacidad Intelectual. Colección FEAPS. Madrid: FEAPS.

WANG, M. M. y KobER, R. R. (2011). Embracing an era of rising family quality of life research. Journal of Intellectual Disability Research, 55 (12), 1093-1097.

Werner, S. S., Edwards, M. M., Baum, N. N., Brown, I. I., Brown, R. I. e Isaacs, B. J. (2009). Family quality of life among families with a member who has an intellectual disability: An exploratory examination of key domains and dimensions of the revised. Journal of Intellectual Disability Research, 53 (6), 501-511.

Wilder, J. y Granlund, M. (2015). Stability and change in sustainability of daily routines and social networks in families of children with profound intellectual and multiple disabilities. Journal of Applied Research in Intellectual Disabilities, 28 (2), 133-144.

Zuna, N., Summers, J. A., Turnbull, A. P., Hu, X. y Xu, S. (2010). Theorizing about family quality of life. En R. KoHer (Ed.), Enhancing the quality of life of people with intellectual disability: From theory to practice (pp. 241-278). Dordrecht: Springer.

Zuna, N. I., Turnbull, A. P. y Summers, J. (2009). Family quality of life: Moving from measurement to application. Journal of Policy E Practice in Intellectual Disabilities, 6 (1), 25-31. 


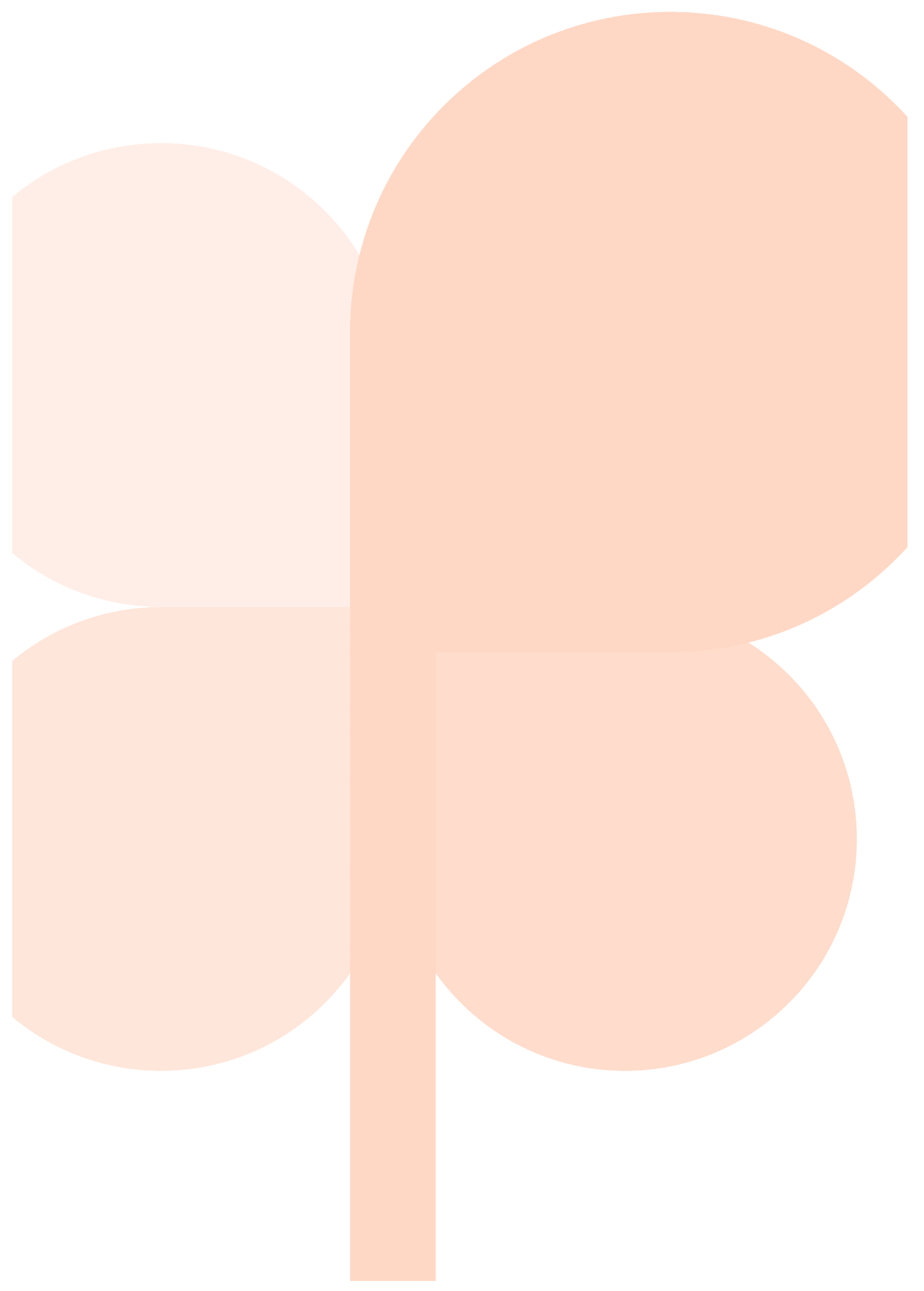

